\title{
Parameterized Complexity of Vertex Deletion into Perfect Graph Classes ${ }^{\star}$
}

\author{
Pinar Heggernes ${ }^{1}$, Pim van 't Hof ${ }^{1}$, Bart M. P. Jansen ${ }^{2}$, \\ Stefan Kratsch ${ }^{2}$, and Yngve Villanger ${ }^{1}$ \\ 1 University of Bergen, P.O. Box 7803, N-5020 Bergen, Norway \\ \{pinar.heggernes,pim.vanthof, yngve.villanger\}@ii.uib.no \\ 2 Utrecht University, P.O. Box 80.089, 3508 TB Utrecht, The Netherlands \\ \{bart,kratsch\}@cs.uu.nl
}

\begin{abstract}
Vertex deletion problems are at the heart of parameterized complexity. For a graph class $\mathcal{F}$, the $\mathcal{F}$-DELETION problem takes as input a graph $G$ and an integer $k$. The question is whether it is possible to delete at most $k$ vertices from $G$ such that the resulting graph belongs to $\mathcal{F}$. Whether PERfect Deletion is fixed-parameter tractable, and whether Chordal Deletion admits a polynomial kernel, when parameterized by $k$, have been stated as open questions in previous work. We show that Perfect Deletion $(k)$ and Weakly Chordal Deletion $(k)$ are $W[2]$-hard. In search of positive results, we study restricted variants such that the deleted vertices must be taken from a specified set $X$, which we parameterize by $|X|$. We show that for PERFECT Deletion and WEAKLY Chordal Deletion, although this restriction immediately ensures fixed parameter tractability, it is not enough to yield polynomial kernels, unless $\mathrm{NP} \subseteq \mathrm{coNP} /$ poly. On the positive side, for Chordal Deletion, the restriction enables us to prove a kernel with $\mathcal{O}\left(|X|^{4}\right)$ vertices.
\end{abstract}

\section{Introduction}

The minimum number of vertices to delete from a given graph so that the resulting graph is a member of a graph class $\mathcal{F}$ is a way of measuring how close the input graph is to being in $\mathcal{F}$. Vertex deletion problems correspond to some of the most fundamental NP-complete graph problems [14]; if we take $\mathcal{F}$ to be the class of complete graphs, we simply get the CLIQUE problem, and if we take $\mathcal{F}$ to be the class of edgeless graphs, we get the VERTEX Cover problem. Vertex deletion problems have received much attention also for more general graph classes $\mathcal{F}$; e.g., the number of vertex deletions needed to make a graph acyclic, corresponding to the FeEdBACK Vertex SeT problem, has applications in deadlock recovery. In addition, seemingly unrelated problems are easier to solve on graphs which are close to being a member of some simple graph class.

\footnotetext{
* Supported by the Netherlands Organization for Scientific Research, project "KERNELS: Combinatorial Analysis of Data Reduction", and by the Research Council of Norway, project "SCOPE: Exploiting Structure to Cope with Hard Problems".
} 
For example, Graph IsOmorphism [18] can be solved efficiently on "almost" forests. Since all vertex deletion problems for non-trivial, polynomial-time recognizable, hereditary graph classes are NP-complete [19], many of these problems have been studied with respect to parameterized complexity [12].

In parameterized complexity we associate with every instance a non-negative integer $k$, called the parameter. A parameterized problem $Q \subseteq \Sigma^{*} \times \mathbb{N}$ is Fixed Parameter Tractable (FPT) if there is an algorithm which decides whether $(x, k) \in$ $Q$ in time $f(k)|x|^{\mathcal{O}(1)}$ for some arbitrary function $f$. There is a hierarchy of intractable parameterized problem classes above FPT, the main ones being: FPT $\subseteq W[1] \subseteq W[2] \subseteq \ldots \subseteq W[P] \subseteq X P$. An important subfield of parameterized complexity is kernelization [17], a formalization of data reduction. For a parameterized problem $Q$, if there is an algorithm which transforms an instance $(x, k)$ in time $(|x|+k)^{\mathcal{O}(1)}$ into an equivalent instance $\left(x^{\prime}, k^{\prime}\right)$, i.e., with the guarantee that $(x, k) \in Q \Leftrightarrow\left(x^{\prime}, k^{\prime}\right) \in Q$ and $\left|x^{\prime}\right|, k^{\prime} \leq g(k)$ for some arbitrary function $g$, then we say that $Q$ admits a kernel. In fact, a parameterized problem is FPT if and only if it is decidable and admits a kernel [12]; unfortunately the guaranteed size is typically exponential. If $g \in k^{\mathcal{O}(1)}$ then the obtained kernel is a polynomial kernel, which is a highly desirable property. Whether or not an FPT problem admits a polynomial kernel has received considerable attention recently, especially after the establishment of methods for proving non-existence of polynomial kernels, up to some complexity theoretical assumptions $[4,3,5]$. Vertex deletion problems are most commonly defined and parameterized as follows.

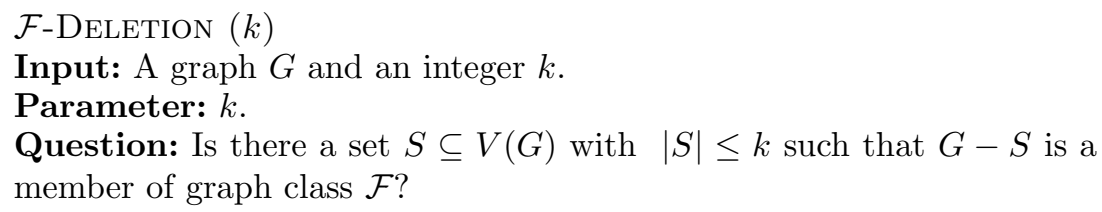

Well known FPT problems of this type include VerTex Cover [9] (i.e., Independent Deletion $(k)$ ), Feedback Vertex Set [25] (i.e., Forest Deletion $(k)$ ), Odd Cycle Transversal [24] (i.e., Bipartite Deletion $(k)$ ), Chordal Deletion $(k)$ [21] and Planar Deletion $(k)$ [22]. When $\mathcal{F}$ is characterized by a finite set of forbidden induced subgraphs, then $\mathcal{F}$-DELETION $(k)$ is FPT [8], and admits a polynomial kernel through its correspondence to $d$ Hitting Set $(k)$ [1]. If $\mathcal{F}$ is characterized by a finite set of forbidden minors, then $\mathcal{F}$-DELETION $(k)$ is FPT with an $\mathcal{O}\left(n^{3}\right)$ time algorithm for every fixed value of $k$, obtained via the Graph Minors machinery [13]. A recent result of Fomin et al. shows that if this set of forbidden minors contains an "onion graph", then the problem additionally admits a polynomial kernel [13].

For some time it was unknown whether there were vertex deletion problems, for hereditary graph classes that can be recognized in polynomial time, that were not FPT by the natural parameterization. This was settled by Lokshtanov, who showed that Wheel-free Deletion $(k)$ is $W[2]$-hard [20]. He posed the FPT-status of Perfect Deletion $(k)$ as an open question.

We show that Perfect Deletion $(k)$ and Weakly Chordal DeleTION $(k)$ are $W[2]$-hard. We then turn our focus to kernelization. The CHORDAL 
Deletion $(k)$ problem was studied by Marx [21], who gave an involved FPT algorithm which combines branching with an irrelevant-vertex reduction step and Courcelle's Theorem for bounded treewidth. He posed as an open question whether Chordal Deletion $(k)$ admits a polynomial kernel. Even an exponential simple kernel would yield a simple FPT algorithm for CHORDAL DeLETION $(k)$ through exploring the kernel by brute force, and hence finding a polynomial kernel seems to be formidable task. Observing that access to an approximate solution is often helpful in designing kernels $[23,6]$, and that no good approximation algorithms for CHORDAL Deletion $(k)$ are known, one might consider the effect of supplying a constant-factor approximation to the kernelization algorithm. This does not help much; an exponential-size kernel which has access to a constant-factor approximation would immediately yield a new algorithm to solve ChORDAl Deletion Compression $(k)$ [16], solving the general version as well. The following restricted problem variant is more amenable to analysis:

Restricted $\mathcal{F}$-Deletion $(|X|)$

Input: A graph $G$, a set of vertices $X \subseteq V(G)$ such that $G-X$ is a member of class $\mathcal{F}$, and an integer $k$.

Parameter: $|X|$.

Question: Is there a set $S \subseteq X$ of size at most $k$ such that graph $G-S$

is a member of class $\mathcal{F}$ ?

In this restricted variant of vertex deletion problems, a set $X$ of candidates for deletion is given in the input, and we are only allowed to delete vertices from $X$. The parameter measures the number of candidate vertices, and with this parameterization the problem becomes trivially FPT. However, we show that Restricted Perfect Deletion $(|X|)$ and Restricted Weakly Chordal DeLetion $(|X|)$ do not admit polynomial kernels unless NP $\subseteq$ coNP/poly, and the same holds for Restricted WheEL-Free Deletion $(|X|)[20]$. In contrast to these hardness results, we show that Restricted Chordal Deletion $(|X|)$ admits a kernel with $\mathcal{O}\left(|X|^{4}\right)$ vertices. We hope that this forms a first step towards a polynomial kernel for Chordal Deletion $(k)$.

Finally, the study of perfect graphs and their subclasses is well established, with several books (e.g., $[7,15])$ and thousands of papers. The interest in the field is boosted by the recent proof of the Perfect Graph Theorem by Chudnovsky et al. [10], after being a conjecture by Berge [2] for over 40 years. Chordal graphs are a subset of weakly chordal graphs that are a subset of perfect graphs.

\section{Preliminaries}

If $G$ is a graph then $V(G)$ and $E(G)$ denote the vertex and edge set, respectively. We only consider finite, simple, and undirected graphs. For a vertex $v \in V(G)$, the set of vertices adjacent to $v$ is called the (open) neighborhood of $v$, and is denoted by $N_{G}(v)$. The closed neighborhood of $v$ is $N_{G}[v]=N_{G}(v) \cup\{v\}$. For a vertex set $S \subseteq V(G)$, the neighborhood of $S$ is $N_{G}(S)=\bigcup_{v \in S} N_{G}(v) \backslash S$. 
The subgraph of $G$ induced by $S$ is denoted by $G[S]$. The graph $G[V(G) \backslash S]$ is denoted by $G-S$. The contraction of an edge $u v \in E(G)$ deletes $u$ and $v$ from $G$ and replaces them with a new vertex whose neighborhood is $N_{G}(\{u, v\})$. The resulting graph is denoted by $G / u v$. For a finite set $X,\left(\begin{array}{l}X \\ a\end{array}\right)$ denotes the collection of all subsets of $X$ of size $a$.

A walk $W$ from vertex $v_{1}$ to vertex $v_{r}$ in a graph $G$ is a sequence of vertices $\left(v_{1}, v_{2}, \ldots, v_{r}\right)$ such that $v_{i} v_{i+1} \in E(G)$ for $1 \leq i \leq r-1$. The vertices $\left\{v_{1}, v_{r}\right\}$ are the endpoints of the walk, whereas $\left\{v_{2}, \ldots, v_{r-1}\right\}$ are the interior vertices of the walk. If $W$ is a walk then we use $V(W)$ to denote the set of its vertices. For a walk $W^{\prime}=\left(x_{1}, \ldots, x_{t}\right)$, we will also use the notation $\left(v_{i}, W^{\prime}, v_{j}\right)$ to denote the walk $\left(v_{i}, x_{1}, \ldots, x_{t}, v_{j}\right)$, assuming that $v_{i} x_{1}, x_{t} v_{j} \in E(G)$. A chord of a walk is an edge between two vertices which are not successive on the walk. A walk without chords is an induced walk. A walk is a path if all its vertices are distinct, and it is a cycle if all interior vertices are distinct and the endpoints coincide.

We denote by $P_{n}$ and $C_{n}$ an induced path and an induced cycle on $n$ vertices, respectively. A hole in a graph is an induced subgraph isomorphic to $C_{t}$ for $t \geq 5$. An anti-hole is the edge-complement of a hole. A hole or anti-hole is odd if it contains an odd number of vertices. A graph is chordal if it does not contain $C_{t}$, for $t \geq 4$, as an induced subgraph; an equivalent condition is that all cycles of length at least four have a chord. A graph is perfect if the chromatic number equals the size of the largest clique for all its induced subgraphs. As conjectured a long time ago [2], and proved recently [10], a graph is perfect if and only if it does not contain any odd hole or odd anti-hole as an induced subgraph. A graph is weakly chordal if it does not contain any hole or anti-hole as an induced subgraph [15]. A cograph is a graph which does not contain $P_{4}$ as an induced subgraph. Weakly chordal graphs, chordal graphs, and cographs are all perfect. In addition, chordal graphs and cographs are both weakly chordal, but they are not related to each other with respect to inclusion (see Fig. 1 in the appendix). It is an easy observation that each of these graph classes is closed under vertex deletions, i.e., hereditary. Furthermore, they can all be recognized in polynomial time.

\section{Hardness of Perfect and Weakly Chordal Deletion}

In this section we prove several hardness results for deleting vertices to obtain a perfect or weakly chordal graph. As the main result we will show that PERFECT Deletion $(k)$ is $W[2]$-hard, by a reduction from Hitting Set $(k)$. We will then argue that the same reduction yields several interesting results as a corollary.

Hitting Set $(k)$

Input: A finite set $U$ of size $n$, a family $\mathcal{H}$ of subsets of $U$, and an integer $k$.

Parameter: $k$.

Question: Is there a set $Y \subseteq U$ of size at most $k$ that has a nonempty intersection with each set of $\mathcal{H}$ ? 
Theorem 1. Perfect Deletion ( $k$ ) is W[2]-hard.

Proof. We give a parameterized reduction from the $W[2]$-complete HitTing Set $(k)$ problem [12]. Let $(U, \mathcal{H}, k)$ be an instance of Hitting Set $(k)$. We assume, without loss of generality, that $|H| \geq 2$ for every $H \in \mathcal{H}$, since sets of size 1 can easily be eliminated in polynomial time. We construct an equivalent instance $(G, k)$ of Perfect Deletion $(k)$, by building a graph $G$ as follows:

- Create an independent set $X$ on $|U|$ vertices; $X=\left\{v_{u} \mid u \in U\right\}$.

- For each set $H=\left\{u_{1}, \ldots, u_{t}\right\} \in \mathcal{H}$, where $t \geq 2$ by assumption, do as follows:

- Add $|H|+1$ new vertices $h_{1}, \ldots, h_{t+1}$ to $G$. The set $\mathcal{G}_{H}=\left\{h_{1}, \ldots, h_{t+1}\right\}$ is called the set gadget for $H$.

- Add the edges $\left\{h_{1}, v_{u_{1}}\right\},\left\{v_{u_{1}}, h_{2}\right\},\left\{h_{2}, v_{u_{2}}\right\}, \ldots,\left\{v_{u_{t}}, h_{t+1}\right\},\left\{h_{t+1}, h_{1}\right\}$, creating an odd chordless cycle $\left(h_{1}, v_{u_{1}}, h_{2}, v_{u_{2}}, \ldots, v_{u_{t}}, h_{t+1}\right)$ of length at least 5 .

- Take the join of the set gadgets by adding all edges between vertices of different set gadgets: for each set $H \in \mathcal{H}$, make all vertices of $\mathcal{G}_{H}$ adjacent to all vertices of $\mathcal{G}_{H^{\prime}}$ for every $H^{\prime} \neq H$.

This concludes the description of the graph $G$. To prove the equivalence of the instances $(U, \mathcal{H}, k)$ and $(G, k)$, we first formulate some claims on the structure of $G$. Claim 1 is proved in the appendix.

Claim 1 The graph $G-X$ is a cograph and therefore perfect.

Claim 2 Any hole in $G$ intersects $X$ and exactly one set gadget $\mathcal{G}_{H}$.

Proof. Since $G-X$ is a cograph by Claim 1 and therefore contains no hole, any hole in $G$ contains at least one vertex of $X$. Moreover, since $X$ is an independent set, the graph $G[X]$ contains no holes. This implies that any hole must intersect at least one set gadget. Since any three vertices from three different set gadgets induce a triangle $K_{3}$, no hole contains vertices from more than two different set gadgets. Now assume for contradiction that $G$ contains a hole $D$ which contains vertices from exactly two set gadgets. Since we have taken the join of the set gadgets, graph $G[V(D) \backslash X]$ is connected and must hence be an induced path. This in turn implies that $D$ contains at most one vertex of $X$, since vertices of the independent set $X$ cannot be consecutive vertices of $D$. However, since holes have at least 5 vertices, this implies that $D$ contains at least 4 consecutive vertices that belong to $G-X$. Since these vertices induce a path on at least 4 vertices, this contradicts the fact that $G-X$ is a cograph by Claim 1 .

Claim 3 Any anti-hole in $G$ has length 5 and is therefore a hole of length 5.

Proof. Recall that an anti-hole in $G$ is a hole in the edge-complement $\bar{G}$ of $G$. Suppose $\bar{G}$ contains a hole $D$. Observe that every set gadget induces a complete graph minus one edge in $\bar{G}$, and that the graphs induced by the set gadgets are exactly the connected components of $\bar{G}-X$. Since a complete graph minus one edge does not contain a hole, $D$ contains at least one vertex of $X$. The set $X$ is a 
clique in $\bar{G}$, so $D$ contains at most two vertices of $X$, which must be consecutive vertices of $D$. This implies that the graph $\bar{G}[V(D) \backslash X]$ is connected, and is therefore contained in one connected component of $\bar{G}-X$; let $\mathcal{G}_{H}$ be the set gadget that induces this connected component. Since $G\left[\mathcal{G}_{H}\right]$ is a complete graph minus one edge in $\bar{G}, D$ contains at most three vertices of $\mathcal{G}_{H}$. This means that $D$ has length at most 5 . Since every hole has length at least 5 by definition, we conclude that any hole in $\bar{G}$, and consequently any anti-hole in $G$, has length exactly 5 . The claim follows from the fact that $C_{5}$ is self-complementary.

Claim 4 If $S \subseteq V(G)$ such that $G-S$ is perfect, then there is a set $S^{\prime} \subseteq X$ with $\left|S^{\prime}\right| \leq|S|$ such that $G-S^{\prime}$ is perfect.

Proof. Let $S \subseteq V(G)$ be a set which intersects all odd holes and odd antiholes in $G$, and assume $S \nsubseteq X$. Consider a vertex $w \in S \backslash X$, which must belong to some set gadget $\mathcal{G}_{H}$. By construction, $w$ has exactly two neighbors in $G\left[X \cup \mathcal{G}_{H}\right]$, and at least one of these is contained in $X$; let $v_{u}$ be such a vertex in $N_{G}(w) \cap X$. Let $D$ be a hole in $G$ containing $w$. As a result of Claim $2, D$ is contained in $G\left[X \cup \mathcal{G}_{H}\right]$. Since $w$ has only two neighbors in $G\left[X \cup \mathcal{G}_{H}\right], D$ also contains vertex $v_{u}$. Hence every hole that contains $w$ also contains $v_{u}$. The same holds for every anti-hole containing $w$, since every anti-hole in $G$ is a hole by Claim 3. This proves that for $S^{\prime \prime}=(S \backslash\{w\}) \cup\left\{v_{u}\right\}$ the graph $G-S^{\prime \prime}$ is also perfect. By repeating this argument we obtain the desired set $S^{\prime} \subseteq X$.

We are now set to prove correctness of our reduction. First assume that $(G, k)$ is a yes-instance of Perfect Deletion $(k)$, and let $S \subseteq V(G)$ be a set of at most $k$ vertices such that $G-S$ is perfect. By Claim 4, we may assume that $S \subseteq X$. Let $Y \subseteq U$ contain all $u \in U$ for which the corresponding vertex $v_{u}$ is contained in $S$. By construction of $G$, for every set $H=\left\{u_{1}, \ldots, u_{t}\right\} \in \mathcal{H}$, the set $\left\{v_{u_{1}}, \ldots, v_{u_{t}}\right\} \cup \mathcal{G}_{H}$ induces an odd hole in $G$. Since $S \subseteq X$ intersects all odd holes, $S$ contains at least one vertex of $v_{u_{1}}, \ldots, v_{u_{t}}$, which shows that $Y$ hits set $H$. Hence $Y$ is a hitting set for $\mathcal{H}$ of the requested size, which means that $(U, \mathcal{H}, k)$ is a yes-instance of Hitting $\operatorname{Set}(k)$.

For the reverse direction, assume that $(U, \mathcal{H}, k)$ is a yes-instance of HitTing SET $(k)$, and let $Y \subseteq U$ be a set of at most $k$ vertices that intersects every set in $\mathcal{H}$. We let $S \subseteq X \subseteq V(G)$ contain all vertices $v_{u}$ for which $u \in Y$. Clearly, $|S| \leq k$; we show that $G-S$ is perfect. By Claim 3, it suffices to check that $S$ contains at least one vertex of every odd hole in $G$. Let $D$ be an odd hole in $G$. By Claim 2, $D$ contains vertices of $X$ and vertices of exactly one set gadget $\mathcal{G}_{H}$, for some $H=\left\{u_{1}, \ldots, u_{t}\right\} \in \mathcal{H}$. Recall that the vertices of $\left\{v_{u_{1}}, \ldots, v_{u_{t}}\right\} \cup \mathcal{G}_{H}$ induce an odd hole in $G\left[X \cup \mathcal{G}_{H}\right]$, and it follows from the construction of $G$ that $G\left[X \cup \mathcal{G}_{H}\right]$ does not contain any other hole. Thus, $D$ is an odd hole constructed to represent some set $H \in \mathcal{H}$. Since $Y \cap H \neq \emptyset$, this implies that $S \cap V(D) \neq \emptyset$ as well. This shows that the two instances are equivalent.

From the construction used in the proof of Theorem 1 it may be verified that all the holes and anti-holes in $G$ are odd: Claim 3 shows that every anti-hole in $G$ is an odd hole, and using Claim 2 the chordless cycles of $G$ can be seen 
to coincide with the odd holes used to represent the sets of $\mathcal{H}$. Hence $G-S$ is perfect if and only if $G-S$ is weakly chordal, and we immediately get the following corollary.

\section{Corollary 1. Weakly Chordal Deletion $(k)$ is $W[2]$-hard.}

For another implication of our reduction, consider the problem HitTing $\operatorname{SET}(n)$, where the parameter is the number of variables $n$. Dom et al. [11] showed that this problem does not admit a polynomial kernel unless NP $\subseteq$ coNP/poly. Claim 4 shows that we may demand the deletion set $S$ of our constructed instance to be a subset of $X$, without changing the answer. Since the reduction of Theorem 1 can be performed in polynomial time, we can also interpret it as a polynomial parameter transformation from an instance $(U, \mathcal{H}, k)$ of HITTING Set $(n)$ with parameter $n=|U|$ to an instance $(G, X, k)$ of RESTRICTED Perfect Deletion $(|X|)$ with parameter $|X|=|U|$ (and also to Restricted Weakly Chordal Deletion $(|X|))$. By standard techniques for kernelization lower bounds $([11])$, this polynomial parameter transformation yields the following results.

Corollary 2. Neither Restricted Perfect Deletion $(|X|)$ nor RestricTed Weakly Chordal Deletion $(|X|)$ admits a polynomial kernel, unless $N P \subseteq \operatorname{coNP} /$ poly.

\section{Polynomial Kernel for Restricted Chordal Deletion}

In this section, we prove that Restricted Chordal Deletion $(|X|)$ admits a kernel with at most $2|X|^{4}+|X|^{3}+|X|^{2}+|X|$ vertices. To simplify the reduction procedure, we first work on an annotated version of the problem, and we show a smaller kernel for the annotated problem. Then we show how to transform an instance of the annotated problem into an instance of RESTRICTED CHORDAL DeLetion $(|X|)$ by slightly increasing the size. The annotated problem is defined after the following proposition that will be used in our proofs.

Proposition 1 ([21]). Let $G$ be a graph containing three vertices $u, v, w$, such that $u, w \in N_{G}(v)$ and $u w \notin E(G)$. If there is a walk $W$ from $u$ to $w$ none of whose interior vertices is in $N_{G}[v]$, then there is a chordless cycle in $G$ containing $\{u, v, w\}$ and a non-empty subset of $V(W)$.

Annotated Restricted Chordal Deletion $(|X|)$

Input: A graph $G$, a set of vertices $X \subseteq V(G)$ such that $G-X$ is chordal, a set of critical pairs $C \subseteq\left(\begin{array}{c}X \\ 2\end{array}\right)$, and an integer $k$.

Parameter: $|X|$.

Question: Is there a set $S \subseteq X$ of size at most $k$ such that $G-S$ is chordal, and $S$ contains at least one vertex of each pair $\{u, v\} \in C$ ? 
A set $S$ as described above is called a valid solution. For ease of notation, we will use $F$ to denote the chordal graph $G-X$. We now present four reduction rules that will constitute a kernelization algorithm for ANNOTATED RESTRICTED Chordal Deletion $(|X|)$. Each reduction rule takes as input an instance of this problem, and if the rule is applicable, it outputs an equivalent reduced instance. We apply the reduction rules in the given order; whenever we apply a reduction rule to an instance, we assume that none of the previous reduction rules can be applied on that instance. For all the rules and proofs below, let $(G, X, C, k)$ be an instance of Annotated Restricted Chordal Deletion $(|X|)$.

Rule 1 If there is a vertex $v \in X$ such that $G[\{v\} \cup V(F)]$ is not chordal, then reduce to the instance $\left(G-\{v\}, X \backslash\{v\}, C^{\prime}, k-1\right)$, where $C^{\prime}$ is obtained from $C$ by deleting all pairs which contain $v$.

Rule 2 If there are two vertices $u, v \in X$ with $\{u, v\} \notin C$, such that $G[\{u, v\} \cup$ $V(F)]$ is not chordal, then reduce to the instance $(G, X, C \cup\{\{u, v\}\}, k)$.

The proofs that Rule 1 and 2 are safe are given in the appendix.

Rule 3 If there is an edge $u v \in E(F)$ such that $N_{G}(u) \cap X=N_{G}(v) \cap X$, then reduce to the instance $(G / u v, X, C, k)$.

Lemma 1. Rule 3 is safe.

Proof. Assume that Rule 1 and Rule 2 are not applicable, whereas Rule 3 is applicable, on $(G, X, C, k)$. Suppose $(G, X, C, k)$ is a yes-instance, and let $S$ be a valid solution. Since $X$ does not contain $u$ or $v$, we have $S \cap\{u, v\}=\emptyset$. Observe that the class of chordal graphs is closed under contracting edges. Hence, since $G-S$ is chordal, $(G-S) / u v=G / u v-S$ is also chordal. Consequently, $S$ is a valid solution for $(G / u v, X, C, k)$, which is thus a yes-instance.

For the reverse direction, suppose that $(G / u v, X, C, k)$ is a yes-instance, and let $S$ be a valid solution. We will show that $S$ is also a valid solution for $(G, X, C, k)$. For contradiction, assume that $G-S$ contains an induced cycle $D$ of length at least 4 . Since $G-X$ is chordal, $D$ contains at least one vertex of $X \backslash S$. In fact, since Rule 1 and Rule 2 cannot be applied and $S$ contains at least one vertex of each critical pair, $D$ contains at least three vertices $x, y, z \in X \backslash S$. If $D$ contains neither $u$ nor $v$, then $D$ was also present in $G / u v-S$, contradicting the assumption that $G / u v-S$ is chordal. If $D$ contains both $u$ and $v$, then $D$ is an induced cycle on at least 5 vertices in $G-S$, containing $u, v, x, y, z$. This means that $G / u v-S$ contains an induced cycle on at least 4 vertices, contradicting the assumption that $G / u v-S$ is chordal.

Consider finally the case where $D$ contains either $u$ or $v$, say $u$. At most two of the vertices $x, y, z$ are adjacent to $u$, as $D$ has no chords. Assume without loss of generality that $y \notin N_{G}(u)$, which implies that $y \notin N_{G}(v)$ since $y \in X$ and $N_{G}(u) \cap X=N_{G}(v) \cap X$. Let $a, b$ be the predecessor and successor of vertex $y$ on $D$, from which it follows that $a b \notin E(G)$. Since $D$ is an induced cycle, it contains a walk from $a$ to $b$ none of whose interior vertices belongs to $N_{G}[y]$. If 
we substitute the occurrence of $u$ on this walk by the vertex resulting from the contraction of $u$ and $v$, we obtain a walk from $a$ to $b$ in $G / u v$ none of whose interior vertices belongs to $N_{G / u v}[y]$, and none of the vertices of this walk are contained in $S$. By Proposition 1 this shows that $G / u v-S$ contains a chordless cycle of length at least 4 , contradicting the assumption that $G / u v-S$ is chordal. We conclude that $G-S$ is chordal, and consequently that $(G, X, C, k)$ is a yesinstance.

With these first three rules, we are able to bound the length of any induced path in $F$.

Lemma 2. If $(G, X, C, k)$ is a reduced instance with respect to Rules 1-3, and $P$ is an induced path in $F$, then $P$ contains at most $2|X|+1$ vertices.

Proof. Suppose $F$ contains an induced path $P=\left(p_{1}, \ldots, p_{t}\right)$. We say that an edge $p_{i} p_{i+1}$ of $P$ is promoted by a vertex $x \in X$ if $x \in N\left(p_{i}\right) \backslash N\left(p_{i+1}\right)$ or $x \in N\left(p_{i+1}\right) \backslash N\left(p_{i}\right)$. For any two consecutive vertices $p_{i}$ and $p_{i+1}$ of $P$, we have that $N\left(p_{i}\right) \cap X \neq N\left(p_{i+1}\right) \cap X$, since Rule 3 cannot be applied. This means in particular that there is a vertex $x \in X$ such that $x \in N\left(p_{i}\right) \backslash N\left(p_{i+1}\right)$ or $x \in N\left(p_{i+1}\right) \backslash N\left(p_{i}\right)$, for each $i$ between 1 and $t-1$. Consequently, every edge of $P$ is promoted by some vertex of $X$. Moreover, if a vertex $x \in X$ is adjacent to two vertices $p_{i}$ and $p_{j}$ of $P$ with $i<j$, then $x$ is also adjacent to each of the vertices $p_{i+1}, \ldots, p_{j-1}$, as otherwise $G[\{x\} \cup V(F)]$ would not be chordal, and Rule 1 would have been applicable. Thus, each vertex of $X$ can promote at most two edges of $P$. Since every edge of $P$ is promoted by some vertex of $X$, it follows that $P$ contains at most $2|X|$ edges, and hence at most $2|X|+1$ vertices.

We now give the final reduction rule that will provide the polynomial bound on the size of a kernel.

Rule 4 Repeat the following for each ordered triple $(u, v, w)$ of distinct vertices in $X$ : if there is an induced path $P$ between $u$ and $w$ whose internal vertices are all in $F-N_{G}(v)$, then mark all the internal vertices of $P$. Let $Y$ be the set of vertices of $F$ that were not marked during this procedure. Reduce to the instance $(G-Y, X, C, k)$.

Lemma 3. Rule 4 is safe.

Proof. Assume that Rules 1-3 cannot be applied, whereas Rule 4 can be applied on $(G, X, C, k)$. Suppose $(G, X, C, k)$ is a yes-instance, and let $S$ be a valid solution. Since the class of chordal graphs is closed under taking induced subgraphs, and $G-S$ is chordal, we see that $G-Y-S$ is chordal. It follows that $S$ is also a valid solution for $(G-Y, X, C, k)$.

For the reverse direction, suppose that $(G-Y, X, C, k)$ is a yes-instance, and let $S$ be a valid solution for this instance. We will show that $S$ is a valid solution for $(G, X, C, k)$ as well. Assume for contradiction that it is not. This means that $G-Y-S$ is chordal, whereas $G-S$ has an induced cycle $D$ of length at least 
4. As we argued in the proof of Rule 3 , since $G-X$ is chordal and Rules 1-2 cannot be applied, $D$ contains at least three vertices $x, y, z \in X \backslash S$. The subgraph $F^{\prime}=F[V(D) \backslash X]$ is a disjoint union of induced paths. If each of the vertices of $F^{\prime}$ was marked during the application of Rule 4, then $D$ is also an induced cycle in $G-Y-S$, which contradicts the assumption that $G-Y-S$ is chordal.

Suppose there is a path $P$ in $F^{\prime}$ which contains an unmarked vertex. Let $x, z \in X$ be the two neighbors on $D$ of the endpoints of $P$. Note that $x$ and $z$ belong to $X \backslash S$, and they are distinct since $D$ contains at least three vertices of $X \backslash S$. Let $y \notin\{x, z\}$ be a third vertex of $D$ which belongs to $X \backslash S$. Since $D$ has no chords, the path $(x, P, z)$ is an induced path from $x$ to $z$ whose interior vertices are contained in $F-N_{G}[y]$. Hence, when we tested the triple $(x, y, z)$ in Rule 4, we found a path $P^{\prime}$ from $x$ to $z$, not containing any neighbor of $y$, all whose vertices we marked. Let $a$ and $b$ be the predecessor and successor of $y$ on $D$, which implies $a b \notin E(G)$. The cycle $D$ contains a walk from $a$ to $b$ none of whose interior vertices belongs to $N_{G}[y]$. If we substitute the occurrence of $P$ on this walk by $P^{\prime}$, we also obtain a walk from $a$ to $b$, and since $P^{\prime}$ does not contain any vertex of $N_{G}[y]$, it follows that none of the interior vertices of this new walk belongs to $N_{G}[y]$. By Proposition 1 this implies that $G$ contains a chordless cycle $D^{\prime}$ on a subset of the vertices of the new walk. By construction the new walk avoids $S$, so the chordless cycle exists in $G-S$. Since the new walk avoids $P$, the cycle $D^{\prime}$ contains strictly fewer of the unmarked vertices than the original cycle $D$. Consequently, after repeating this procedure at most $|D|$ times, we find an induced cycle $D^{\prime \prime}$ in $G-S$ containing no unmarked vertex of $F$, and at least four vertices: $x, y, z$ and a marked vertex of $F$. Since all the vertices of $V\left(D^{\prime \prime}\right) \cap V(F)$ are marked, $D^{\prime \prime}$ is also an induced cycle in $G-Y-S$, which contradicts the assumption that $G-Y-S$ is chordal.

We are now ready to state the kernel result on the annotated problem.

Theorem 2. Annotated Restricted Chordal Deletion $(|X|)$ admits a kernel with at most $2|X|^{4}+|X|^{3}+|X|$ vertices.

Proof. Rules 1-3 can trivially be applied in polynomial time. When we apply Rule 4, we need to test $\mathcal{O}\left(|X|^{3}\right)$ triples. For each triple $(u, v, w)$, determining whether there is a path $P$ from $u$ to $w$ whose internal vertices are contained in $F-N_{G}(v)$ can be done by simply trying to find a shortest path from $u$ to $w$ in the subgraph of $G$ induced by $u, w$ and the vertices of $F-N_{G}(v)$. Hence this rule can also be applied in polynomial time.

Let $(G, X, C, k)$ be an instance that is reduced with respect to Rules 1-4. Observe that $G[F]$ can be covered by $|X|^{3}$ induced paths, and by Lemma 2, each such path contains at most $2|X|+1$ vertices. Consequently, $|V(F)| \leq$ $2|X|^{4}+|X|^{3}$. Since $V(G)=V(F) \cup X$, the result follows.

Finally, the main result of this section is given below, whereas its proof is moved to the appendix.

Theorem 3. Restricted ChORdal Deletion $(|X|)$ admits a kernel with at most $2|X|^{4}+|X|^{3}+|X|^{2}+|X|$ vertices. 


\section{Conclusion}

The reduction in the proof of Theorem 1 shows that it is possible to construct a small set of vertices $X$ which models the universe of a Hitting SeT instance, and that for every subset $X^{\prime} \subseteq X$ we can add some vertices to create a hole in the graph which intersects $X$ exactly in $X^{\prime}$, without creating other holes. This makes it possible to reduce Hitting Set $(k)$ to Perfect Deletion $(k)$, while also giving a reduction from Hitting Set $(n)$ to Restricted Perfect DeleTion $(|X|)$. Our positive result for Restricted Chordal Deletion $(|X|)$ shows that chordless cycles, the forbidden structures for chordal graphs, do not have the same modeling power.

It will be very interesting to settle the kernelization complexity of CHORDAL Deletion $(k)$. We observe that this problem admits a linear-vertex kernel on planar and bounded-genus graphs: this follows from the meta-theorem by Bodlaender et al. [4], since the problem can be formulated in MSOL, has finite integer index, and is quasi-compact because chordal planar graphs have constant treewidth. Concerning structural parameterizations, it is not hard to prove that Chordal Deletion admits a polynomial kernel when parameterized by the size of a minimum vertex cover. A slightly more involved construction shows that the same good news holds for the parameterization by the size of a minimum feedback vertex set. As an intermediate step in obtaining a polynomial kernel for Chordal Deletion $(k)$, one might consider Chordal Deletion parameterized by vertex deletion distance to an interval graph.

We conclude with some open questions regarding graph modification problems. It would be interesting to determine the FPT status of Perfect Edge Deletion/Completion. Since perfect graphs are closed under edge-complement, the deletion and completion problems are equally hard. The question of INTERVAL Vertex Deletion has been open for some time. Maybe a study of ATFree Vertex Deletion might shed some insight into this problem. Besides computing these modification sets, using them as parameters to other problems is also an interesting area which has not been thoroughly explored. For example, what is the status of FEEDBACK VERTEX SET parameterized by vertex deletion distance to a chordal graph, or INDUCED LONG PATH with the same parameter? These parameterizations have the potential to "beat treewidth", since a graph of large treewidth can nevertheless be close to chordal.

Acknowledgments. We would like to thank Dániel Marx for an insightful discussion on CHORDAL DELETION.

\section{References}

1. F. N. Abu-Khzam. A kernelization algorithm for d-Hitting set. J. Comput. Syst. Sci., 76(7):524-531, 2010.

2. C. Berge. Färbung von graphen, deren sämtliche bzw. deren ungerade kreise starr sind. Wiss. Z. Martin-Luther-Univ. Halle-Wittenberg Math.-Natur. Reihe, 10:114, 1961. 
3. H. L. Bodlaender, R. G. Downey, M. R. Fellows, and D. Hermelin. On problems without polynomial kernels. In Proc. ICALP 2008, pages 563-574, 2008.

4. H. L. Bodlaender, F. V. Fomin, D. Lokshtanov, E. Penninkx, S. Saurabh, and D. M. Thilikos. (Meta) Kernelization. In Proc. 50th FOCS, pages 629-638, 2009.

5. H. L. Bodlaender, S. Thomassé, and A. Yeo. Kernel bounds for disjoint cycles and disjoint paths. In Proc. ESA 2009, pages 635-646, 2009.

6. H. L. Bodlaender and T. C. van Dijk. A cubic kernel for feedback vertex set and loop cutset. Theory Comput. Syst., 46(3):566-597, 2010.

7. A. Brandstädt, V. B. Le, and J. P. Spinrad. Graph classes: a survey. Society for Industrial and Applied Mathematics, Philadelphia, PA, USA, 1999.

8. L. Cai. Fixed-parameter tractability of graph modification problems for hereditary properties. Inf. Process. Lett., 58(4):171-176, 1996.

9. J. Chen, I. A. Kanj, and G. Xia. Improved upper bounds for vertex cover. Theoretical Comput. Sci., 411:3736-3756, 2010.

10. M. Chudnovsky, N. Robertson, P. Seymour, and R. Thomas. The strong perfect graph theorem. Annals of Mathematics, 164:51-229, 2006.

11. M. Dom, D. Lokshtanov, and S. Saurabh. Incompressibility through colors and IDs. In Proc. 36th ICALP, pages 378-389, 2009.

12. R. Downey and M. R. Fellows. Parameterized Complexity. Monographs in Computer Science. Springer, New York, 1999.

13. F. V. Fomin, D. Lokshtanov, N. Misra, G. Philip, and S. Saurabh. Hitting forbidden minors: Approximation and kernelization. In Proc. 28th STACS, pages 189-200, 2011.

14. M. R. Garey and D. S. Johnson. Computers and Intractability. W. H. Freeman and Co., 1978.

15. M. C. Golumbic. Algorithmic Graph Theory and Perfect Graphs, volume 57 of Annals of Discrete Mathematics. North-Holland Publishing Co., 2004.

16. J. Guo, H. Moser, and R. Niedermeier. Iterative compression for exactly solving NP-hard minimization problems. In Algorithmics of Large and Complex Networks, pages 65-80, 2009.

17. J. Guo and R. Niedermeier. Invitation to data reduction and problem kernelization. SIGACT News, 38(1):31-45, 2007.

18. S. Kratsch and P. Schweitzer. Isomorphism for graphs of bounded feedback vertex set number. In Proc. 12th SWAT, pages 81-92, 2010.

19. J. M. Lewis and M. Yannakakis. The node-deletion problem for hereditary properties is NP-complete. J. Comput. Syst. Sci., 20(2):219-230, 1980.

20. D. Lokshtanov. Wheel-free deletion is W[2]-hard. In Proc. 3rd IWPEC, pages 141-147, 2008.

21. D. Marx. Chordal deletion is fixed-parameter tractable. Algorithmica, 57(4):747768, 2010.

22. D. Marx and I. Schlotter. Obtaining a planar graph by vertex deletion. In Proc. 33rd $W G$, pages 292-303, 2007.

23. H. Moser. A problem kernelization for graph packing. In Proc. 35th SOFSEM, pages 401-412, 2009.

24. B. A. Reed, K. Smith, and A. Vetta. Finding odd cycle transversals. Oper. Res. Lett., 32(4):299-301, 2004.

25. S. Thomassé. A quadratic kernel for feedback vertex set. ACM Transactions on Algorithms, 6(2), 2010. 


\section{Appendix: Omitted proofs and a figure}

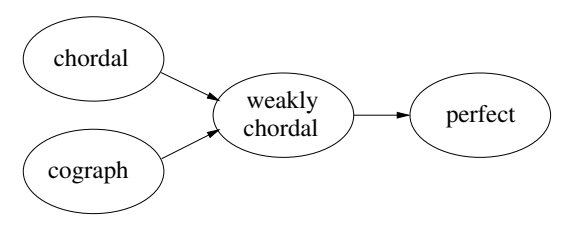

Fig. 1. The inclusion relationship between the graph classes mentioned in this paper. The $\rightarrow$ represents the $\subset$ relation between the classes.

Proof of Claim 1. Observe that for each set $H \in \mathcal{H}$ the graph $G\left[\mathcal{G}_{H}\right]$ is a disjoint union of complete graphs on one or two vertices; hence this graph is $P_{4^{-}}$ free and therefore a cograph. The graph $G-X$ is the join of all the graphs $G\left[\mathcal{G}_{H}\right]$ for $H \in \mathcal{H}$, and since it is well-known [7] that the join of cographs is also a cograph, this proves the claim.

Lemma 4. Rule 1 is safe.

Proof. Assume that Rule 1 is applicable on $(G, X, C, k)$. Suppose that $(G-$ $\left.\{v\}, X \backslash\{v\}, C^{\prime}, k-1\right)$ is a yes-instance, and let $S^{\prime}$ be a valid solution for this instance. Then clearly $S^{\prime} \cup\{v\}$ is a valid solution for $(G, X, C, k)$. For the other direction, suppose that $(G, X, C, k)$ is a yes-instance, and let $S$ be a valid solution for $(G, X, C, k)$. Since $G[\{v\} \cup V(F)]$ is not chordal, we must have $v \in S$. This implies that deleting the set $S^{\prime}=S-\{v\}$ from the graph $G-\{v\}$ yields a chordal graph. Since $S^{\prime}$ has size at most $k-1,\left(G-\{v\}, X \backslash\{v\}, C^{\prime}, k-1\right)$ is a yes-instance.

Lemma 5. Rule 2 is safe.

Proof. Assume that Rule 2 is applicable on $(G, X, C, k)$, and let $C^{\prime}=C \cup$ $\{\{u, v\}\}$. If $\left(G, X, C^{\prime}, k\right)$ is a yes-instance, then clearly $(G, X, C, k)$ is a yesinstance, since $C$ is a proper subset of $C^{\prime}$. For the other direction, suppose that $(G, X, C, k)$ is a yes-instance, and let $S$ be a valid solution. Since $G[\{u, v\} \cup V(F)]$ is not chordal, we must have $S \cap\{u, v\} \neq \emptyset$. Hence $S$ contains at least one vertex of every pair in $C^{\prime}$, and $\left(G, X, C^{\prime}, k\right)$ is a yes-instance.

Proof of Theorem 3. Given an instance $(G, X, C, k)$ of Annotated ReStricted Chordal Deletion $(|X|)$, we show how to compute in polynomial time an equivalent instance $\left(G^{\prime}, X, k\right)$ of Restricted Chordal DeleTION $(|X|)$ such that $\left|V\left(G^{\prime}\right)\right| \leq|V(G)|+|X|^{2}$, from which the result follows. 
Let $(G, X, C, k)$ be an instance of Annotated Restricted Chordal DeleTION $(|X|)$. We create a graph $G^{\prime}$ from $G$ as follows. For each pair $\{u, v\} \in C$, we add two new vertices $u^{\prime}, v^{\prime}$ to $G$, and edges $u u^{\prime}, u v^{\prime}, v u^{\prime}, v v^{\prime}$ if $u v \notin E(G)$, and edges $u u^{\prime}, u^{\prime} v^{\prime}, v^{\prime} v$ if $u v \in E(G)$. Note that $G^{\prime}-X$ is chordal. Let $\mathcal{C}$ denote the collection of edge-disjoint copies of $C_{4}$, one for each pair in $C$, that are created this way. Suppose $(G, X, C, k)$ is a yes-instance of Annotated Restricted Chordal Deletion $(|X|)$, and let $S \subseteq X$ be of size at most $k$ such that $G-S$ is chordal and $S$ contains at least one vertex of every pair in $C$. Let $x$ and $y$ be two vertices in $G^{\prime}$ such that $\{x, y\} \in C$. Since $S$ contains at least one of the vertices $x$ and $y$, the vertices $x^{\prime}$ and $y^{\prime}$, that were added for this pair, are not part of any cycle in $G^{\prime}-S$. This, together with the assumption that $G-S$ is chordal, implies that $G^{\prime}-S$ is chordal. Consequently, $\left(G^{\prime}, X, k\right)$ is a yes-instance of Restricted Chordal Deletion $(|X|)$. For the reverse direction, suppose that $\left(G^{\prime}, X, k\right)$ is a yes-instance of Restricted Chordal Deletion $(|X|)$, and let $S \subseteq X$ be of size at most $k$ such that $G^{\prime}-S$ is chordal. This means in particular that $G-S$ is chordal, since $G-S$ is an induced subgraph of $G^{\prime}-S$. Since $G^{\prime}-S$ is chordal, $S$ contains at least one vertex of every induced $C_{4}$ in $\mathcal{C}$. For every cycle $D \in \mathcal{C}$ with $V(D)=\left\{x, x^{\prime}, y, y^{\prime}\right\}$ and $\{x, y\} \in C$, we have that $X \cap\left\{x^{\prime}, y^{\prime}\right\}=\emptyset$, and hence $S \cap\left\{x^{\prime}, y^{\prime}\right\}=\emptyset$. Consequently, $S$ contains at least one vertex of the critical pair $\{x, y\} \in C$, and $(G, X, C, k)$ is a yes-instance of Annotated Restricted Chordal Deletion $(|X|)$. Since we added 2 vertices for each pair in $C$, we have $\left|V\left(G^{\prime}\right)\right| \leq|V(G)|+2\left|\left(\begin{array}{c}|X| \\ 2\end{array}\right)\right| \leq|V(G)|+|X|^{2}$. 